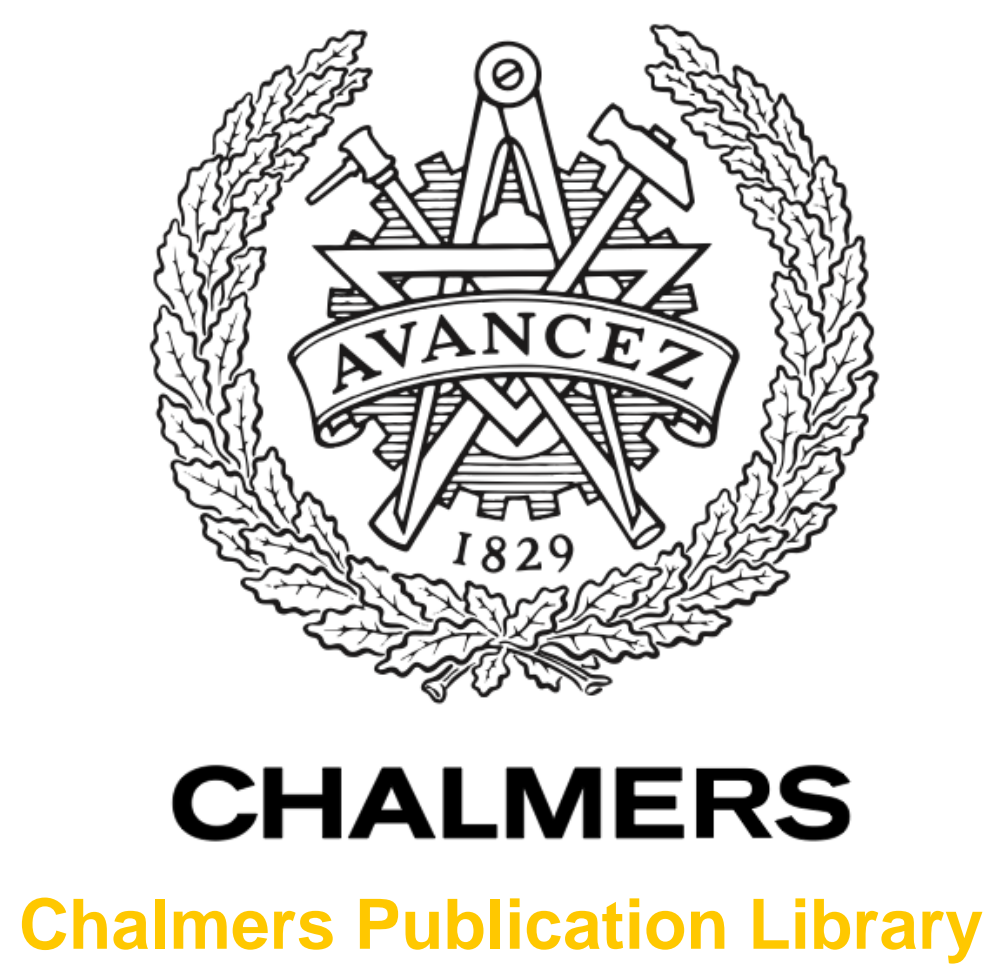

\title{
Screening of external magnetic perturbation fields due to sheared plasma flow
}

This document has been downloaded from Chalmers Publication Library (CPL). It is the author's version of a work that was accepted for publication in:

Nuclear Fusion (ISSN: 0029-5515)

Citation for the published paper:

Li, L. ; Liu, Y. ; Liang, Y. et al. (2016) "Screening of external magnetic perturbation fields due to sheared plasma flow". Nuclear Fusion, vol. 56(9),

http://dx.doi.org/10.1088/0029-5515/56/9/092008

Downloaded from: http://publications.lib.chalmers.se/publication/243705

Notice: Changes introduced as a result of publishing processes such as copy-editing and formatting may not be reflected in this document. For a definitive version of this work, please refer to the published source. Please note that access to the published version might require a subscription.

Chalmers Publication Library (CPL) offers the possibility of retrieving research publications produced at Chalmers University of Technology. It covers all types of publications: articles, dissertations, licentiate theses, masters theses, conference papers, reports etc. Since 2006 it is the official tool for Chalmers official publication statistics. To ensure that Chalmers research results are disseminated as widely as possible, an Open Access Policy has been adopted.

The CPL service is administrated and maintained by Chalmers Library. 


\title{
Screening of external magnetic perturbation fields due to sheared plasma flow
}

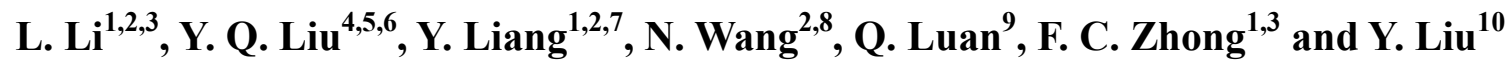 \\ ${ }^{1}$ College of Science, Donghua University, Shanghai 201620, China \\ ${ }^{2}$ Forschungszentrum Jülich GmbH, Institut für Energie- und Klimaforschung - Plasmaphysik, Jülich, Germany \\ ${ }^{3}$ Member of Magnetic Confinement Fusion Research Centre, Ministry of Education, China \\ ${ }^{4}$ CCFE, Culham Science Centre, Abingdon, OX14 3DB, UK \\ ${ }^{5}$ Southwestern Institute of Physics, PO Box 432, Chengdu 610041, China \\ ${ }^{6}$ Department of Earth and Space Science, Chalmers University of Technology, SE-412 96 Gothenburg, Sweden \\ ${ }^{7}$ Institute of Plasma Physics, Chinese Academy of Sciences, P.O. Box 1126, Hefei230031, China \\ ${ }^{8}$ State Key Laboratory of Advanced Electromagnetic Engineering and Technology, Huazhong University of Science and \\ Technology, Wuhan, Hubei 430074, China \\ ${ }^{9}$ School of Mathematical and Physical Sciences, Dalian University of Technology, Panjin 124221, China \\ ${ }^{10}$ Key Laboratory of Materials Modification by Laser, Ion and Electron Beams (Ministry of Education), School of Physics \\ and Optoelectronic Technology, Dalian University of Technology, Dalian 116024, China
}

*E-mail of corresponding authors: 1.li@fz-juelich.de; Yueqiang.Liu@ukaea.uk; y.liang@fz-juelich.de

\begin{abstract}
Within the single fluid resistive magneto-hydrodynamic (MHD) model, systematic toroidal modelling efforts are devoted to investigate the plasma response induced screening of the applied external 3D magnetic field perturbations in the presence of sheared toroidal flow. One particular issue of interest is addressed, when the local flow speed approaches zero at the perturbation rational surface inside the plasma. Subtle screening physics, associated with the favourable averaged toroidal curvature effect (the GGJ effect [Glasser A H et al 1975 Phys. Fluids 7 875]), is found to play an essential role at slow flow near the rational surface by enhancing the screening at reduced flow. A strong cancellation effect between different terms of the Ohm's law is discovered, leading to different screening physics in the GGJ regime, as compared to that of the conventional screening of the typical resistive-inertial regime occurring at faster flow. These modelling results may be applicable to interpret certain mode locking experiments, as well as type-I edge localized mode suppression experiments, with
\end{abstract}


resonant magnetic field perturbations being applied to tokamak plasmas at low input toroidal torque.

\section{Introduction}

The plasma response to external magnetic field plays an important role in understanding various three-dimensional (3D) physics phenomena relevant to tokamak plasmas, such as the resonant field amplification (RFA) [1-5], the active control of the resistive wall mode (RWM) with magnetic feedback [6-11], the mode locking $[12,13]$ and the error field correction [1416], as well as the suppression or mitigation of the edge localized modes (ELMs) in H-mode plasmas using resonant magnetic perturbations (RMP)[17-20].

One key element of the plasma response to $3 \mathrm{D}$ fields is the screening of the magnetic pitch aligned resonant components. Perfect screening occurs if the plasma is assumed to be ideal (i.e. with vanishing resistivity), which is often the case in modelling the RFA and the RWM control experiments for high beta (high temperature) plasmas, where the plasma pressure often exceeds the no-wall Troyon beta limit for the ideal kink instability [21]. On the other hand, only partial screening occurs in a resistive plasma, which is often the adopted model for simulating tokamak experiments at lower beta. This is particularly relevant for the mode locking experiments due to field errors, as well as the ELM control experiments using RMP fields. Understanding the field screening phenomena in these low beta (below the Troyon limit) resistive plasmas is the primary objective of the present work.

In a resistive plasma, the screening effect, or eventually the screening current generated inside the plasma, is a combined result of both the plasma conductivity and the plasma flow. Significant theory and modelling efforts have been devoted to study the plasma response induced screening. Early work is often developed within the cylindrical approximation, and applied mainly to the tearing mode (TM) locking [12, 13, 22], as well as the ELM control 
related RMP problems [6, 23]. During recent years, significant modelling efforts have been carried out to study the plasma response to the 3D RMP fields in toroidal geometry [24-32].

It is now well established that the detailed screening physics, associated with the plasma flow, depends on the plasma model assumptions. In particular, the single fluid model predicts a strong screening due to the toroidal flow of thermal ions $[13,24]$, whilst the two fluid theory predicts screening due to the perpendicular (to the magnetic field lines) flow of thermal electrons [26, 27, 33]. In the limit of vanishing speed of the respective flow (at rational surfaces), both models would predict full penetration of the external resonant fields.

However, little modelling work has been performed, in order to understand how the screening changes, as the plasma flow gradually approaches zero near the perturbation rational surface inside the plasma, and how the flow shear affects the results. This is of particular interest following the recent realization that, according to the single fluid theory, the favorable averaged toroidal curvature effect at very slow flow which is often associated with the TM stabilization in a toroidal plasma, provides additional screening of the external fields [34]. This work provides the first systematic, numerical investigation of this so called GGJ-screening regime using the MARS-F code [35]. Comparison is also made with the conventional resistive-inertial (RI) regime [13], which occurs at faster flow than the GGJ regime. The results may be relevant for interpreting not only the mode locking experiments, but also the ELM control (in particularly the ELM suppression) experiments using RMP fields at low input toroidal torque.

We shall only consider the single fluid model in this study. The electron flow screening physics may be particularly relevant for studying the RMP penetration problem [Waelbroeck NF 52, 074004 (2012)], where the primary interest is in the H-mode pedestal region. The electron diamagnetic flow is normally large in the pedestal region. In this work, we are 
mainly interested in the flow screening for the core TM (at the $q=2$ surface here), where the electron diamagnetic flow contribution is normally not significant. Even for the typical RMP problem, it seems that the single fluid approximation, including the toroidal flow induced plasma response screening, works satisfactorily well when compared with experiments [19, $25,36]$. This can be partially explained by the fact that the conventional single fluid flow screening and the perpendicular electron flow screening are essentially equivalent when casted in the proper mathematical formulation, as discussed in Ref. [16].

Section 2 briefly describes the MARS-F single fluid, resistive plasma response model, and the associated GGJ physics. Section 3 introduces three families of radial profiles for the toroidal rotation frequency of the plasma. Systematic rotation scan study is based on these flow profiles. Section 4 reports the computational results using the first two families of the flow profiles, both assuming unidirectional flow across the whole plasma minor radius. Section 5 reports results with the third family of flow profiles, where we allow the flow reversal near the rational surface. Peculiar screening physics is discovered and studied in this section. Section 6 draws conclusion and discussion.

\section{The toroidal, single fluid, resistive plasma response model, and the GGJ effects}

We used the MARS-F code [35] to compute the plasma response to the external magnetic field under the DC condition, while prescribing a radial profile for the plasma flow speed $\mathbf{V}_{0}=R \Omega \hat{\phi}$, where $R$ is the plasma major radius, $\Omega$ is the angular frequency of the toroidal

rotation, specified as a function of the plasma minor radius, and $\hat{\phi}$ is the unit vector along the geometric toroidal angle $\phi$. MARS-F solves the linearized single fluid, resistive, full MHD equations in a general toroidal geometry.

$$
i\left(\Omega_{R M P}+n \Omega\right) \xi=\mathbf{v}+(\xi \cdot \nabla \Omega) R \hat{\phi}
$$




$$
\begin{aligned}
& i \rho\left(\Omega_{R M P}+n \Omega\right) \mathbf{v}=-\nabla p+\mathbf{j} \times \mathbf{B}+\mathbf{J} \times \mathbf{b}-\rho[2 \Omega \hat{Z} \times \mathbf{v}+(\mathbf{v} \cdot \nabla \Omega) R \hat{\phi}] \\
& i\left(\Omega_{R M P}+n \Omega\right) \mathbf{b}=\nabla \times(\mathbf{v} \times \mathbf{B}-\eta \mathbf{j})+(\mathbf{b} \cdot \nabla \Omega) R \hat{\phi} \\
& i\left(\Omega_{R M P}+n \Omega\right) p=-\mathbf{v} \cdot \nabla P-\Gamma P \nabla \cdot \mathbf{v} \\
& \mathbf{j}=\nabla \times \mathbf{b}
\end{aligned}
$$

where the variables $\xi, \mathbf{v}, p, \mathbf{j}$ and $\mathbf{b}$ denote the plasma displacement, perturbed velocity, pressure, current and magnetic field, respectively. The equilibrium plasma density, magnetic field, pressure and current are denoted by $\rho, \mathbf{B}, P$ and $\mathbf{J}$, respectively. $n$ is the toroidal harmonic number. We consider $n=1$ in this study. The equations are written in dimensionless form, with the length normalized to the major radius of the magnetic axis, the time to the toroidal Alfvén time $\tau_{A}$, the magnetic field to the on-axis vacuum toroidal field $B_{0}$, and the pressure to $B_{0}^{2} / \mu_{0}$. The toroidal rotation frequency $\Omega$ is normalized to the Alfvén frequency $\Omega_{A}$.

The external 3D field is generated by the currents on the coils which are set in vacuum. In the MARS-F code, the coil current density $\mathbf{j}_{R M P}$, as a source term, is prescribed which satisfies Ampere's law $\nabla \times \mathbf{b}=\mathbf{j}_{R M P}$. The frequency of the source current is denoted by $\Omega_{R M P}$ in Eqs. (1-4). This term vanishes if dc coil currents are assumed, as in this study. This is often the case for field errors as well as for the RMP fields in the ELM control experiments.

The above equations are solved in an equilibrium magnetic flux surface based straight field line coordinate system, where the toroidal angle is chosen as the geometric angle. The minor radius is labelled by the equilibrium poloidal flux function. The poloidal angle is 
chosen such that the Jacobian, associated with the transformation from the cylindrical coordinates, is proportional to $R^{2}$.

The GGJ effect, first theoretically investigated for general toroidal configurations [37], is essentially associated with the favorable average magnetic curvature of a toroidal equilibrium, and with the presence of finite equilibrium pressure gradient near the mode rational surface. The effect is important only inside the resistive layer. The key consequence of this effect is the modification of the so called "internal" tearing index, which is often used to match the external tearing index from the ideal region, in order to obtain the growth rates for resistive instabilities. More specifically, a new additive term appears in the tearing index associated with the inner layer. This term, being proportional to the resistive interchange index (eventually proportional to the local equilibrium pressure at the rational surface), is inversely proportional to the (complex) frequency of the mode. As a result, the GGJ effect predicts the existence of overstable tearing modes. In this work, however, we shall not be investigating the tearing mode stability. We shall instead study the resistive plasma response to external 3D fields, for a toroidal equilibrium with finite pressure gradient at the rational surface. The same GGJ physics apply, but appearing as a screening effect for the resonant component of the radial magnetic field perturbation, as will be elucidated in this study.

\section{Specification of the plasma equilibrium and flow profiles}

To facilitate understanding of the numerical results from the MARS-F runs, we shall consider a simple equilibrium, which is the same as that assumed in Ref. 34. This equilibrium has a single rational surface associated with the $n=1$ perturbation inside the plasma. The radial profiles of the equilibrium plasma pressure and the safety factor are shown in figure 1(a) and 1(b), respectively. The radial coordinate is defined as $s \equiv \sqrt{\psi_{n}}$, with $\psi_{n}$ being the normalized poloidal flux. The GGJ screening effect, occurring at slow flow, is essentially due to the finite 
equilibrium pressure gradient at the $q=2$ surface. Note that our choice of the equilibrium pressure here is not representative for H-mode plasmas (no pressure pedestal near the plasma edge). The physics effect that we investigate in this work, however, is essentially outside the pedestal region, and thus being valid independent of the existence of a pedestal in the equilibrium pressure.
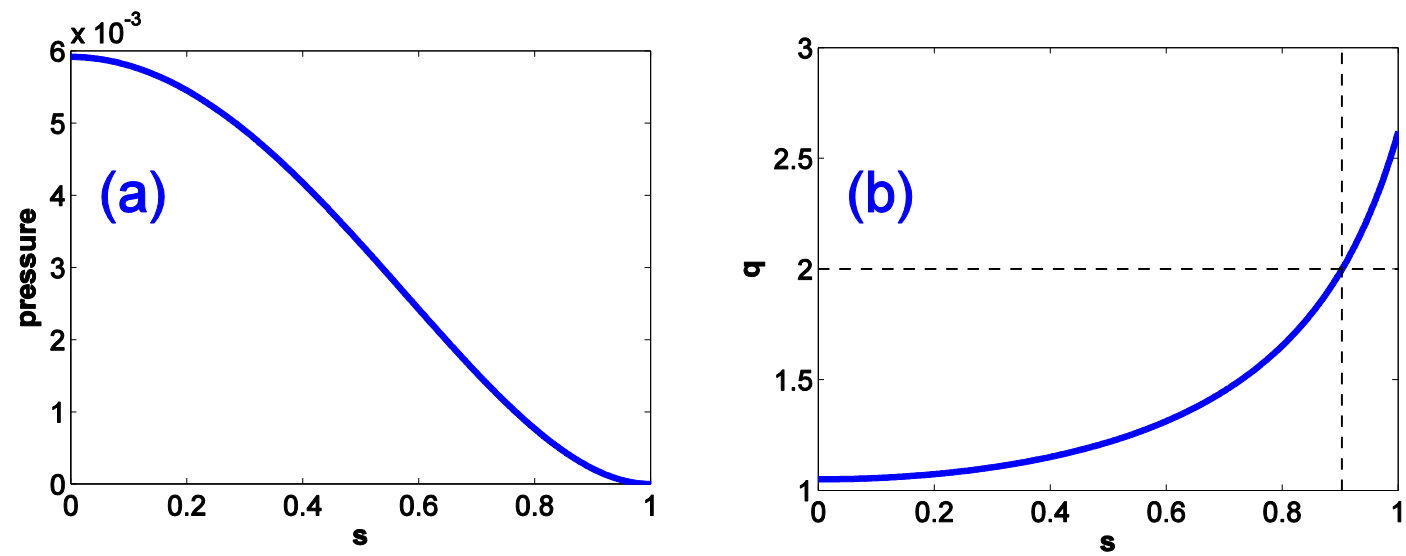

Figure 1. Radial profiles of (a) the plasma equilibrium pressure normalized by $B_{0}^{2} / \mu_{0}$, and (b) the safety factor. The radial coordinate is the square root of the normalized equilibrium poloidal flux. The vertical dash line in (b) denotes the location of the rational $q=2$ surface.

All results in Ref. 34 were obtained assuming a uniform equilibrium rotation profile. With the primary objective of this work to study the effect of the local flow speed at the rational surface on the plasma screening of the external 3D fields, we wish to understand: (i) what happens if the local flow speed vanishes near or at the rational surface? (ii) what is the role played by the local flow shear as well as the global flow profiles? (iii) what is the difference in the screening as the flow speed approaches zero without or with the reversal of the flow direction?

In order to answer the these questions, we consider various ways of specifying the flow profiles, taking special care of the local variation of the flow profile near the rational surface. 
More specifically, we choose three families of sheared plasma rotation frequency profiles as shown below.

\subsection{Model A: A unidirectional flow with global variation of flow profile}

Such a profile is prescribed by the following model

$\Omega=\left(\Omega_{0}-\Omega_{1}\right)\left(1-2 s^{2}+s^{3}\right)+\Omega_{1}$

where $\Omega_{0}$ is the amplitude of the plasma rotation frequency at the magnetic axis, and $\Omega_{1}$ is defined as the rotation frequency at the plasma surface [24]. An example of such a profile, normalized to unity at the magnetic axis, is shown in figure 2(a). Similar to the magnetic shear, we define the flow shear as

$S_{\Omega}=s(d \Omega / d s) / \Omega$

Note that if $\Omega_{1}$ vanishes in Eq. (6), the above defined flow shear is a constant while varying the flow amplitude. In numerical computations, we normally assume a very small edge flow $\Omega_{1}$. Thus the local flow shear is kept nearly constant, while varying the global flow profile by adjusting the flow amplitude.

\subsection{Model B: A unidirectional flow with local variation of flow amplitude}

In order to investigate the relative effects of flow shear and flow amplitude at the rational surface, on the plasma response to the external magnetic field, we consider a new family of profiles, with nearly vanishing plasma flow speed at the rational surface. The profile is defined as

$$
\Omega=\left|\frac{\Omega_{0}}{-2 s_{q}{ }^{2}+s_{q}{ }^{3}}\left(2 s^{2}-s^{3}-2 s_{q}{ }^{2}+s_{q}{ }^{3}\right)\right|\left[1-e^{-\left((q-2) / 10^{-3}\right)^{2}}\right]+\Omega_{q}
$$


where $s_{q}$ is the radial position of the rational surface $q=2$, and $\Omega_{q}$ is the plasma rotation frequency at this surface. The model allows a large variation of $\Omega_{q}$, by orders of magnitude, without significant change of the core rotation $\Omega_{0}$. In addition, the local flow shear at the rational surface vanishes. Figure 2(b) shows one example of the radial equilibrium plasma rotation frequency profile, where $\Omega$ is again normalized into unity at the magnetic axis $\Omega_{0}$.
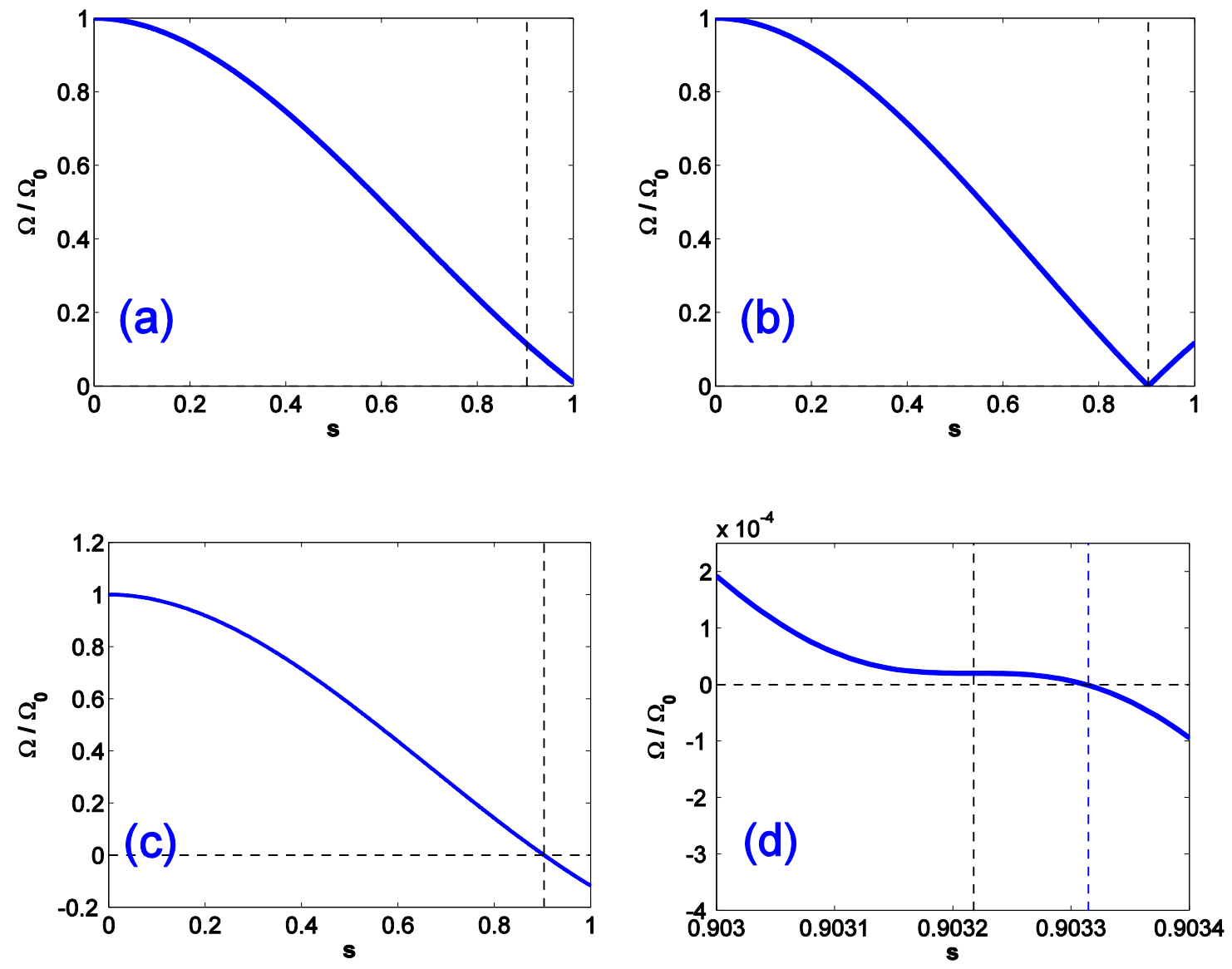

Figure 2. Three types of radial profiles for the equilibrium toroidal rotation frequency of the plasma: (a) without, and (b) with, a strong local variation of the flow profile near the $q=2$ rational surface, indicated by black dashed vertical lines, (c-d) with reversal of the flow direction. (d) shows the details near the rational surface. The blue vertical dash line indicates the position of the vanishing flow surface.

Compared to the global flow model as described in subsection 3.1, the above model allows more flexibility in independent variation of the on-axis flow amplitude and the local 
flow amplitude at the rational surface, at the same time without introducing the flow reversal effect that is described by the next model.

\subsection{Model C: A flow profile with local reversal of flow direction near the rational surface}

As shall be shown later in this work, the local reversal of the flow direction introduces a subtle degeneracy into the screening physics (the Ohm's law), when the zero-crossing coincides with the radial location of the rational surface. Numerically we investigate the screening in this peculiar situation, by radially separating the zero-crossing surface for the flow and the rational surface. We then gradually decrease the distance between these two surfaces.

The flow model is thus proposed as follows

$$
\Omega=\frac{\Omega_{0}}{-2 s_{q}{ }^{2}+s_{q}{ }^{3}}\left(2 s^{2}-s^{3}-2 s_{q}{ }^{2}+s_{q}^{3}\right)\left[1-e^{-((q-2) / D)^{2}}\right]+\Omega_{q}
$$

where the definitions of $s_{q}, \Omega_{q}$ are the same as in Model B. At fixed rotation frequencies on the magnetic axis $\left(\Omega_{0}\right)$ and at the rational surface $\left(\Omega_{q}\right)$, parameter $D$ controls the distance between the flow reversal surface $(\Omega=0)$ and the rational surface $(q=2)$. Taking $D=10^{-3}$ for example, Fig. 2(c-d) show the radial rotation profile with definition (8), with figure 2(d) showing the detailed profile near the rational surface. The flow frequency is again normalized to unity on the magnetic axis. In further computations, the on-axis rotation frequency $\Omega_{0}$ is also one of the systematically varied parameters.

We note that such a choice of the flow profile (8) again yields vanishing flow shear at the rational surface. Compared to a case without the exponential factor in (8), this choice allows a larger radial separation between the zero-crossing surface for the flow and the rational surface, while reducing the local flow amplitude at the rational surface. As a result, it becomes easier 
to isolate the two effects, associated with the distance between the two surfaces on one hand, and the local flow speed at the rational surface on the other hand. The fact that the local flow shear becomes zero is not essential in understanding the screening physics, since as will be shown in the following, the local flow shear does not play a significant role in the screening of the GGJ regime.

Note also that the specification of Model C bears close similarity to that of Model B, allowing comparison between different models. In both Models B and C, the on-axis flow speed $\Omega_{0}$ and that at the rational surface $\Omega_{q}$ can be independently varied, whilst this is not possible with the flow profile Model A, where the flow speeds on the axis and at the rational surface always change in proportion.

\section{Screening due to unidirectional toroidal flow}

We have carried out extensive modeling and analysis efforts, in order to identify the screening physics associated with the local variation of the plasma flow speed near the rational surface. The results, computed by the MARS-F code, are summarized in figure 3, showing plasma response amplitude at the $q=2$ surface, for the $m / n=2 / 1$ resonant harmonic of the perturbed radial field, while scanning the local flow amplitude $\Omega_{q=2}$ at the rational surface. The radial component of the perturbed magnetic field $\mathbf{b}$ is defined as $b^{1}=q(d \psi / d s)(\mathbf{b} \cdot \nabla s) /(\mathbf{B} \cdot \nabla \phi)$. The total field $b_{t o t}^{1}$ produced by both the external coil currents and the perturbed currents of the plasma due to the plasma response. $b_{v a c}^{1}$ is the free-space vacuum field generated by the external coil currents alone, in the absence of the plasma response. 

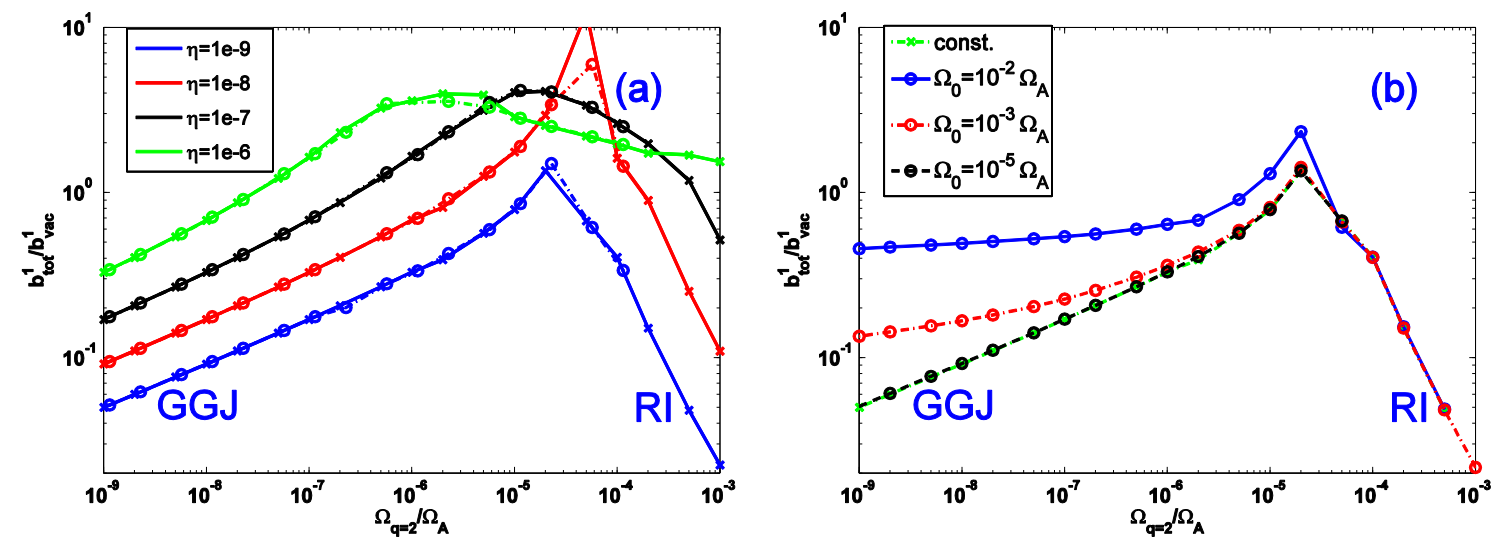

Figure 3. Comparison of the flow screening effects between the uniform flow profile and (a) the flow profile Model A, (b) the flow profile Model B. Plotted is the amplitude of the resonant $(m / n=2 / 1)$ radial field component including the resistive plasma response, normalized by that of the vacuum field, versus the local flow speed at the $q=2$ surface. Various values of the normalized plasma resistivity, of constant radial profile, are assumed in (a), where the results with the uniform flow (lines with crosses) are compared with that of Model A flow profile (lines with circles). Various on-axis flow amplitudes, $\Omega_{0}=10^{-2} \Omega_{A}, \Omega_{0}=10^{-3} \Omega_{A}$ and $\Omega_{0}=10^{-6} \Omega_{A}$, respectively, are chosen in (b) for the flow profile Model B, at the normalized plasma resistivity $\eta=10^{-8}$.

Two flow profile models (Model A and B) are used in Fig. 3. Fig. 3(a) compares the plasma response behavior assuming either a uniform flow profile, or the profile Model A at fixed small edge flow speed of $\Omega_{1}=10^{-2} \Omega_{0}$, with different choices of the plasma resistivity. The resistivity parameter $\eta$ is defined as a dimensionless number by normalizing the physics quantity to a factor $\mu_{0} R_{0} v_{A} / A^{2}$, with $v_{A}=B_{0} / \sqrt{\mu_{0} \rho_{0}}, A$ being the aspect ratio and $\rho_{0}$ the onaxis plasma density. Effectively $\eta$ is the inverse of the Lundquist number. Here a uniform resistivity profile is assumed along the plasma minor radius.

It is interesting to note that the flow profile model A, which has a finite local flow shear at the rational surface, produces nearly the same screening as that by the uniform flow, indicating that the local flow shear is not important in producing the screening effect. In other words, it is the local flow amplitude that quantitatively determines the resonant field screening. This holds for both screening regimes shown in Fig. 3(a) - the GGJ regime at slow 
flow and the RI regime at fast flow. Note that in the GGJ-regime, the response field amplitude decreases (i.e. screening is enhanced) with decreasing plasma flow speed, whilst the opposite occurs in the RI-regime, where the enhanced screening is achieved by increasing the flow speed. The plasma resistivity is another key factor affecting the screening. For both regimes, increasing the plasma resistivity generally reduces the screening.

Model A does not allow strong variation of the local flow profile near the rational surface. A subtle difference appears when such a strong local variation is indeed allowed, as in Model B (Fig. 2(b)). The computed plasma response using this type of flow profile is summarized in Fig. 3(b). Because of the possibility of independently varying the flow speed on the magnetic axis $\left(\Omega_{0}\right)$ and at the rational surface $\left(\Omega_{q=2}\right)$ with Model $\mathrm{B}$, we choose three different values of $\Omega_{0}$ while scanning $\Omega_{q=2}$. The results are also compared with that of the uniform flow profile. As expected, both the GGJ and the RI screening regimes are again obtained, at slow and fast flow at the rational surface, respectively. An interesting observation is that the separation between these two regimes occurs at the same $\Omega_{q=2}$ value, which is about $2 \times 10^{-5} \Omega_{A}$ in our case, independent of the on-axis flow speed $\Omega_{0}$. This again points to the critical role played by the local flow amplitude, on the plasma response induced screening of the resonant field component.

However, the on-axis flow speed, or in other words the global flow profile, does affect the GGJ screening regime as well. Generally at a fixed rational surface flow, increasing the on-axis flow speed reduces the GGJ screening effect. Interestingly, no such effect is seen for the RI screening regime. In order to better understand these two screening regimes, we perform a detailed investigation of the key physics elements that determine the screening. 
The flow screening is essentially described by the radial component of Ohm's law, Eq. (3). In a generic straight field line magnetic coordinate system $(s, \chi, \phi)$, where $\chi$ is the generalized poloidal angle, the radial projection of Eq. (3) can be written as

$$
i\left(\Omega_{R M P}+n \Omega\right) b^{1}=\frac{\partial}{\partial \chi}\left(\psi^{\prime} v^{1}\right)+\frac{\partial}{\partial \phi}\left(\psi^{\prime} q v^{1}\right)-\frac{\partial}{\partial \chi}\left(\eta j_{3}\right)+\frac{\partial}{\partial \phi}\left(\eta j_{2}\right),
$$

where $v^{1}$ is the radial component of the perturbed velocity, defined as $v^{1}=\mathbf{v} \cdot \nabla s ; j_{2}$ and $j_{3}$ are the covariant components of the perturbed plasma current density $\mathbf{j}=j_{1} \nabla s+j_{2} \nabla \chi+j_{3} \nabla \phi$; $\psi^{\prime}=d \psi / d s$. Due to the obvious relation below, derived from Eq. (1) between the radial displacement $\xi^{1}=\xi \cdot \nabla s$ and the perturbed radial velocity

$i\left(\Omega_{R M P}+n \Omega\right) J \xi^{1}=J v^{1}$,

the above radial component of Ohm's law can be further simplified for each individual resonant Fourier harmonic ( $m, n)$, assuming static RMP fields, to

$$
\Omega b_{m n}^{1}=i \psi^{\prime}(m-n q) \Omega \xi_{m n}^{1}+\eta\left[\frac{J B}{\psi^{\prime}} \delta J_{\|}\right]_{m n}
$$

where $b_{m n}^{1}$ is the Fourier harmonic of the radial field $b^{1}$ as defined before, $\xi_{m n}^{1}$ is the Fourier harmonic of the radial displacement $\xi^{1} . J$ is the Jacobian of the coordinate transformation. $B$ is the equilibrium magnetic field amplitude. $\delta J_{\|}$is the parallel (to the equilibrium field line) component of the perturbed plasma current, calculated as

$$
\delta J_{\|}=\frac{\psi^{\prime}}{J B}\left(j_{2}+q j_{3}\right)
$$

Representation (9) greatly simplifies the physics analysis of the flow screening effect, without compromising the toroidal coupling effect. Three terms are identified in Eq. (9): the 
left hand side $(L H S)$ term, $\Omega b_{m n}^{1}$, is related to the radial component of the perturbed magnetic field, indicating the plasma response in terms of the total field; the first right hand side $\left(R H S_{1}\right)$ term, $i \psi^{\prime}(m-n q) \Omega \xi_{m n}^{1}$, is associated with induction, and thus further referred to as the induction term, the second right hand side $\left(R H S_{2}\right)$ term, $\eta\left[J B / \psi^{\prime} \delta J_{\|}\right]_{m n}$, is the resistive term in Ohm's law. The eventual amplitude of the plasma response field, i.e. the screening effect, is the combined result of both RHS terms in Ohm's law.

Figure 4 compares all the key physics components, participating into Ohm's law, between the RI regime and the GGJ regime. For two examples based on the flow profile Model A. Similar results are obtained using the flow Model B. The radial distributions of all three terms of Eq. (9), just near the $q=2$ rational surface, are plotted in Fig. 4(a-d). In the GGJ screening regime (right panels), a large cancellation is observed outside the rational surface, between the induction term and the resistive term in Ohm's law. It is indeed because of this cancellation, the perturbed radial field is small across the whole resistive layer, resulting in the so called GGJ screening.

On the other hand, no such cancellation occurs in the RI regime (left panels). In this case, the major contribution to the plasma response near the rational surface comes from the resistive term $\mathrm{RHS}_{2}$.
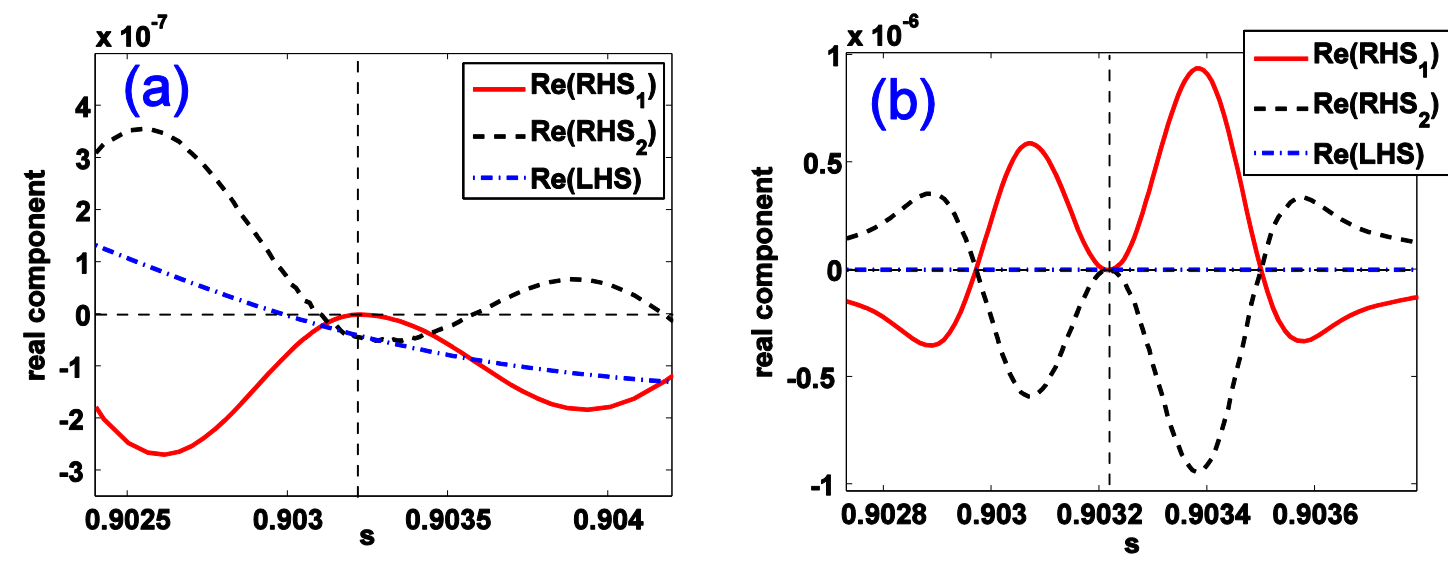

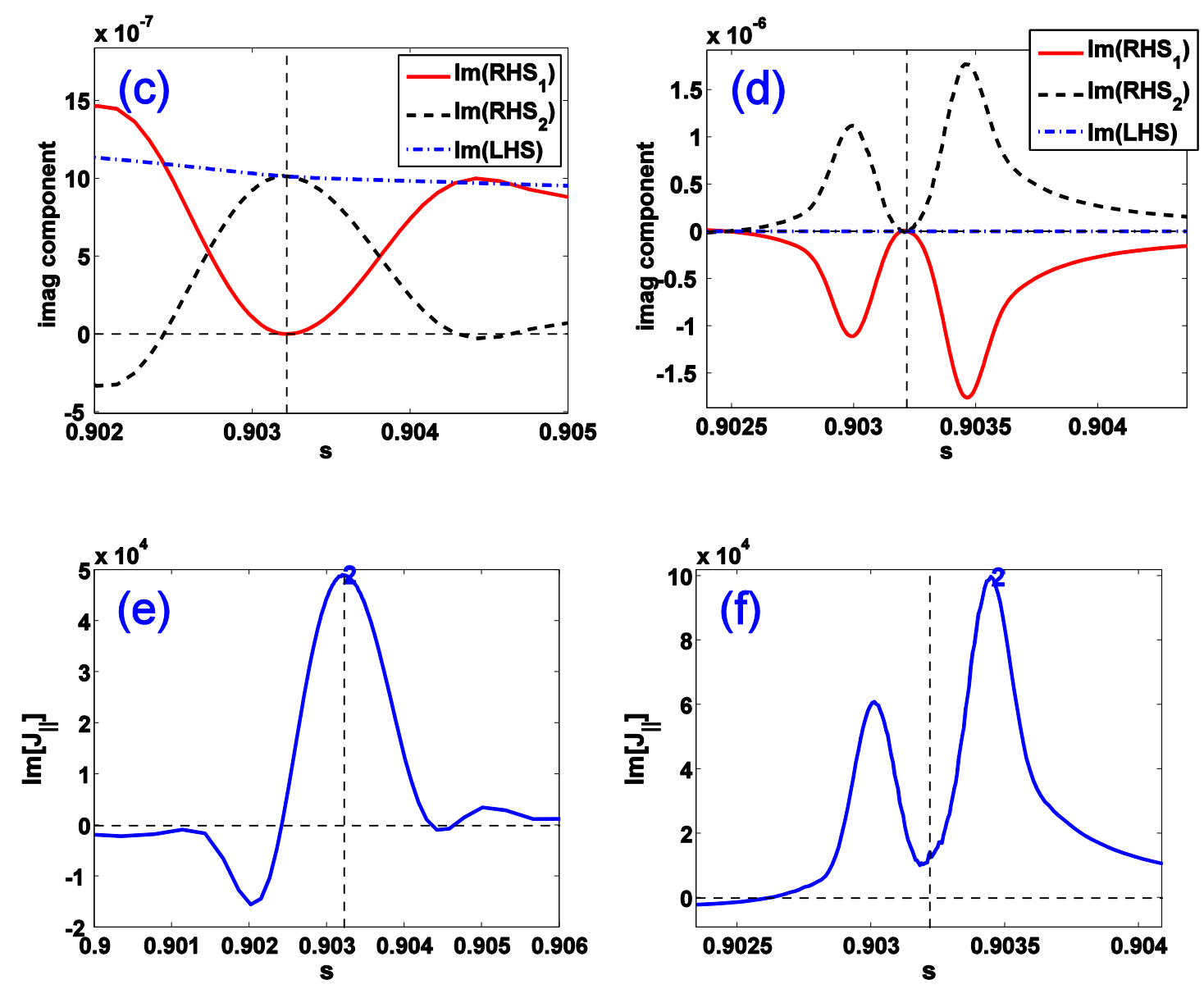

Figure 4. Comparison of key perturbed physics quantities associated with screening, between the RI-regime at fast plasma flow ( $\Omega_{0}=10^{-3} \Omega_{A}, \Omega_{q=2}=10^{-4} \Omega_{A}$, left panels) and the GGJ-regime at slow flow ( $\Omega_{0}=10^{-6} \Omega_{A}$, $\Omega_{q=2}=10^{-7} \Omega_{A}$, right panels). The radial profiles, near the $q=2$ rational surface indicated by vertical dashed lines, are compared for $(a, b)$ the real, and (c, d) the imaginary, parts of three terms in Eq. (9), representing the screening contributions from the induction term $\left(\mathrm{RHS}_{1}\right)$ and the resistive term $\left(\mathrm{RHS}_{2}\right)$ in Ohm's law, and their sum (LHS), and (e, f) the (dominant) imaginary parts of the plasma response generated, $m=2$ perturbed parallel currents, Eq. (10). The flow profile Model B is assumed, with normalized plasma resistivity of $\eta=10^{-8}$.

Since the screening is eventually associated with the plasma generated current perturbation, we also compare the resonant component of the parallel current as defined in Eq. (10). This is shown in Fig. 4(e-f). In the conventional screening regime (figure 4(e)), the perturbed parallel current peak is at the rational surface, providing the screening of the externally applied resonant fields. In the GGJ regime (figure 4(f)), however, the perturbed parallel current peaks are off (but very close to) the rational surface. It is eventually these two off-resonant peaks that provide the screening of the external magnetic field in the GGJ regime. 


\section{Screening due to toroidal flow with local reversal of direction near rational surface}

The plasma response is more subtle when the toroidal flow locally switches direction near the rational surface. Numerically we find that a strong singularity occurs in the response solution near the rational surface, as the flow speed crosses zero, switching sign, exactly at the rational surface. No such singular behavior in the response solution is observed with the flow Model B, as the local flow speed approaches zero exactly at the rational surface.

We attribute the singular solution, associated with the Model $\mathrm{C}$, to the degeneracy of the Ohm's law in Eq. (9). Indeed in the case of $\Omega$ approaching zero at the rational surface, the coefficients in the three terms, associated with the perturbed quantities in Eq. (9) (radial field, radial displacement, and parallel plasma current, respectively), approach zero with different orders. This gives constraints on the possible radial distribution near the rational surface, for the perturbed quantities which have to be self-consistently determined (i.e. satisfying all other MHD equations). This can lead to different possibilities for the response solution. The flow Model B based response solution, for instance, resolves the degeneracy in the equilibrium coefficients by the vanishing parallel current perturbation at the rational surface (at finite resistivity $\eta$ ). The self-consistent response solution based on the flow Model $\mathrm{C}$, on the other hand, does not seem to follow the same cancellation rule. As a result, a stronger singularity develops in the solution near the rational surface, which we believe is un-physical, and cannot be numerically resolved.

However, we still have the possibility to study the flow screening in this case, by two means. First, we separate the resonant surface and the surface where the flow vanishes (further referred to as the zero-flow surface), and gradually decrease the radial distance between these two surfaces. Secondly, we consider a resistivity model that also vanishes at the rational surface 
$\eta=\eta_{0} \min \left[1,\left(\Omega / \Omega_{q}\right)^{4}\right]$

Such a model, to certain degree, removes the degeneracy of Eq. (9).

Figure 5 summarizes the computed response results, following the aforementioned procedures. Since the radial distance between the rational surface and the zero-flow surface is controlled by the model parameter $D$ in Eq. (8), we show two cases (a) and (b), representing roughly two extreme cases: case (a), with $D=5 \times 10^{-3}$, corresponds to the situation where these two surfaces are well decoupled, whilst case (b), with $D=10^{-3}$, shows the results when the physics effects at these two surfaces start to strongly couple to each other.
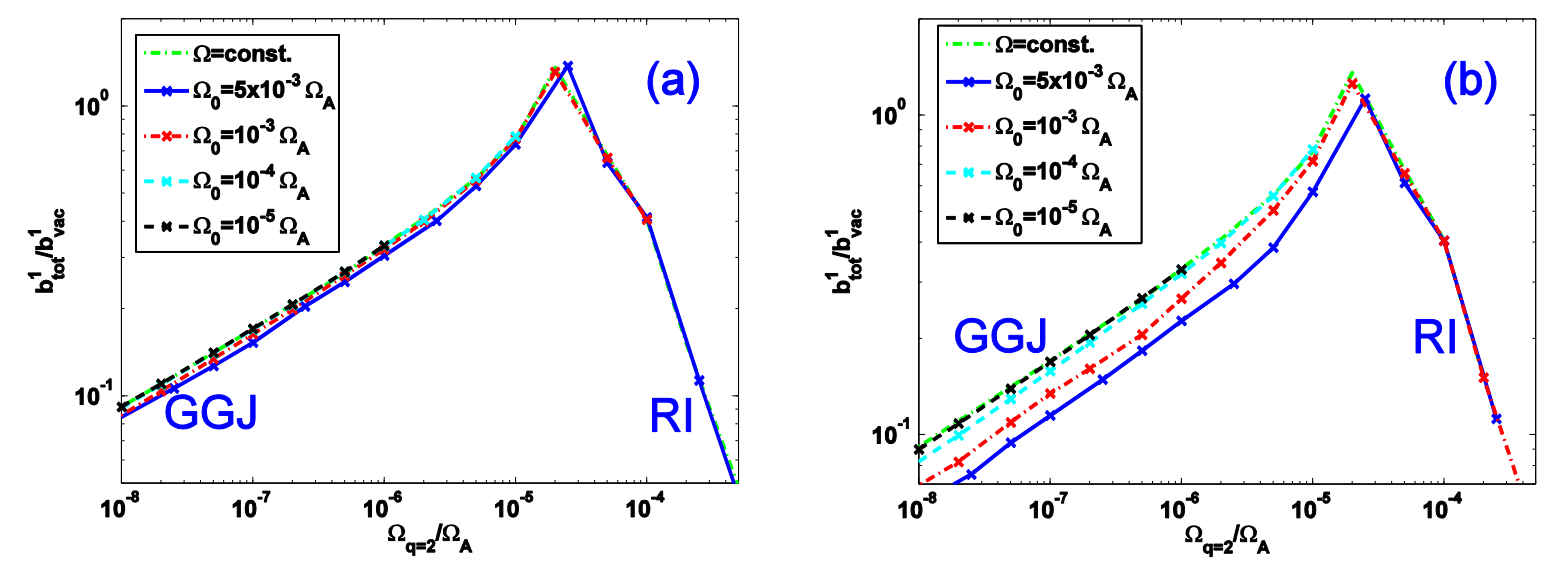

Figure 5. Comparison of the flow screening effects between the uniform flow profile and the flow profile Model C with two choices of the value for the model parameter $D:$ (a) $D=5 \times 10^{-3}$, and (b) $D=10^{-3}$. Plotted is the amplitude of the resonant $(m / n=2 / 1)$ radial field component including the resistive plasma response, normalized by that of the vacuum field, versus the local flow speed at the $q=2$ surface. Different on-axis flow speeds are chosen here in Model $\mathrm{C}$, with $\Omega_{0}=5 \times 10^{-3}, \Omega_{0}=10^{-3}, \Omega_{0}=10^{-4}, \Omega_{0}=10^{-5}$, respectively. The normalized plasma resistivity is fixed at $\eta=10^{-8}$.

First, we note that all these subtle physics have almost no effect on the RI screening regime. They do, however, affect the GGJ regime, although the effect is not as significant as that by the flow Model B. Compared to the uniform flow results, the decoupling between the rational surface and the zero-flow surface results in minor modification of the GGJ screening, 
as shown by Fig. 5(a). On the other hand, the strong coupling effects between the two surfaces do change the GGJ screening as shown in Fig. 5(b). More interestingly, the screening becomes even stronger than the uniform flow case, as the on-axis flow speed is increased. This is opposite to the results with the flow Model B, where the GGJ screening effect is always weaker than the uniform flow case.

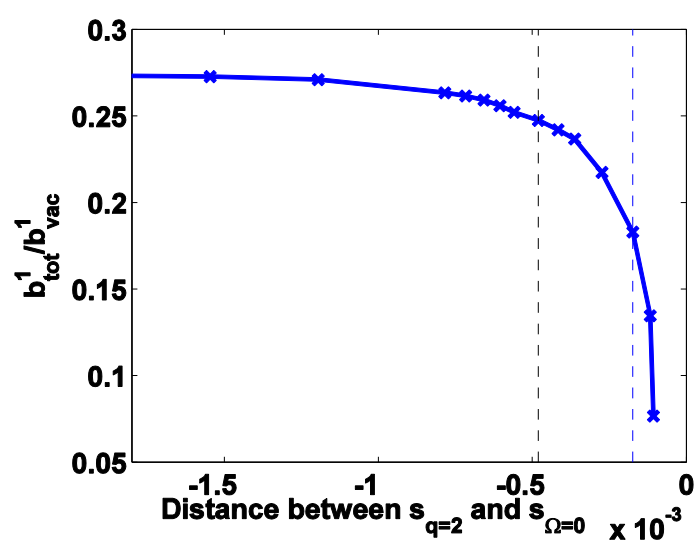

Figure 6. The amplitude of the resonant $(m / n=2 / 1)$ radial field component including the resistive plasma response, computed assuming the flow profile Model $\mathrm{C}$ and normalized by that of the vacuum field, plotted versus the distance between the rational $q=2$ surface and the vanishing flow surface. This surface is controlled by varying the model parameter $D$, with the black and blue vertical dashed lines corresponding to $D=5 \times 10^{-3}$ and $D=10^{-3}$, respectively. Fixed are the on-axis flow speed $\Omega_{0}=5 \times 10^{-3} \Omega_{A}$, the flow at the rational surface $\Omega_{q=2}=5 \times 10^{-7} \Omega_{A}$, as well as the normalized plasma resistivity $\eta=10^{-8}$.

In order to demonstrate the sensitive dependence of the flow Model C based GGJ screening on the separation between the rational surface and the zero-flow surface, Fig. 6 shows the computed resonant response field amplitude versus the radial distance (normalized by the plasma minor radius) between these two surfaces, while scanning the parameter $D$ in Eq. (8) using the flow profile Model C. The response amplitude stays nearly constant as the zero-flow surface is located far away from the rational surface. As the two surfaces radially merge, the plasma response is significantly reduced, resulting in enhanced GGJ screening by the flow reversal near the rational surface. 

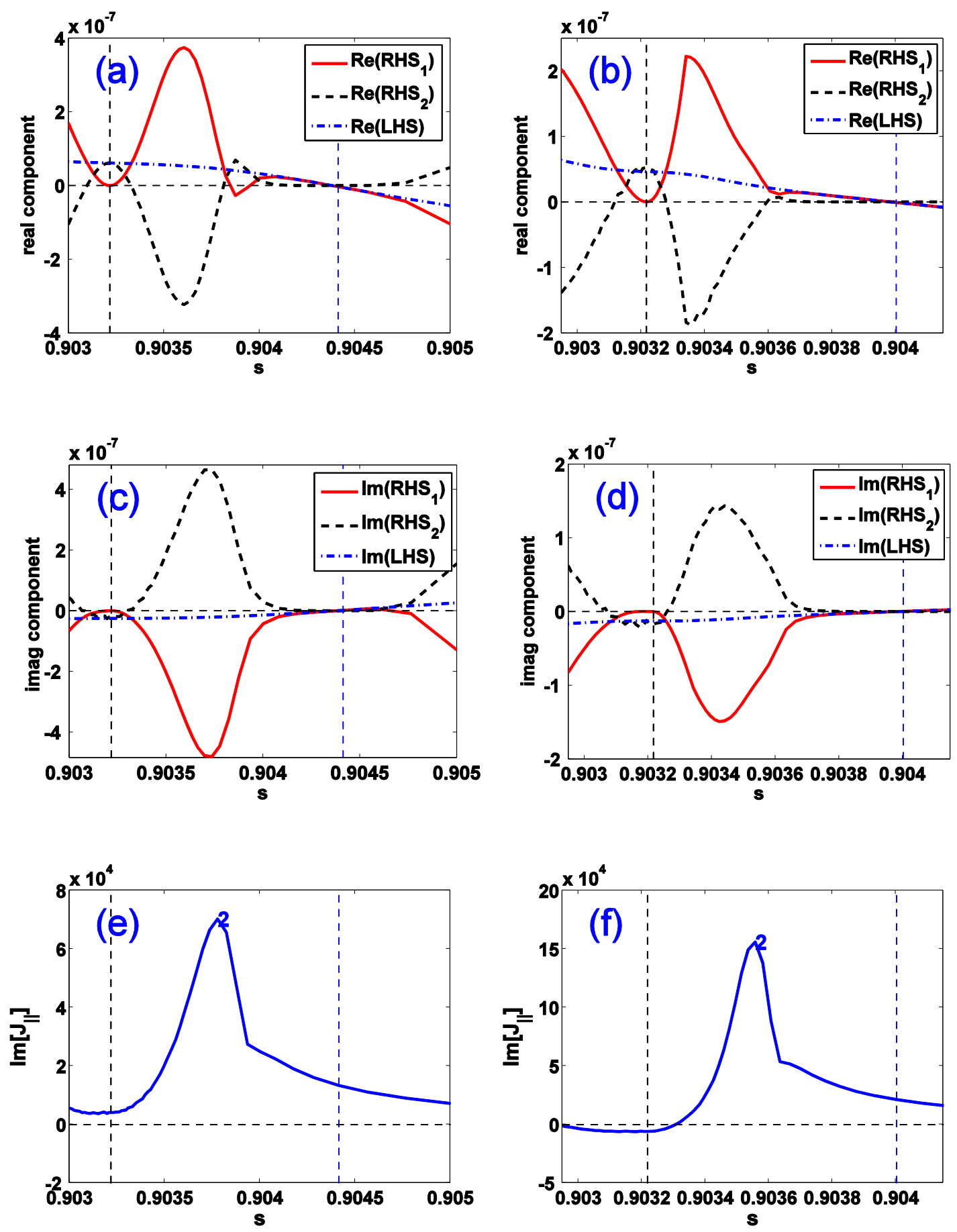

Figure 7. The key perturbed physics quantities associated with screening, computed using the flow profile Model C with two choices of the value for the model parameter $D: D=5 \times 10^{-3}$ (left panels) and $D=10^{-3}$ (right panels). The radial profiles near the $q=2$ rational surface are compared for (a,b) the real, and (c, d) the imaginary, parts of three terms in Eq. (9), representing the screening contributions from the induction term (RHS $)_{1}$ and the resistive term $\left(\mathrm{RHS}_{2}\right)$ in Ohm's law, and their sum (LHS), and (e, f) the (dominant) imaginary parts of the plasma response generated, $m=2$ perturbed parallel currents, Eq. (10). The black (blue) vertical dash line indicates the radial location of the rational $q=2$ (the vanishing flow) surface. Fixed are the on-axis flow speed 
$\Omega_{0}=5 \times 10^{-3} \Omega_{A}$, the flow speed at the rational surface $\Omega_{q=2}=5 \times 10^{-6} \Omega_{A}$, as well as the normalized plasma resistivity $\eta=10^{-8}$.

Figure 7 compares the radial distribution of the key quantities participating in the screening physics, assuming the flow profile Model $\mathrm{C}$ without (a, c, e) and with (b, d, f) strong coupling effects between the rational surface and the zero-flow surface. The radial distributions of the induction and resistive terms are somewhat similar. There is still a large cancellation between the inductive and the resistive terms in these two cases, both being in the GGJ screening regime. However, the cancellation is less complete than that of the flow profile Model B case. The significant difference between the decoupled surfaces and the coupled surfaces is the amplitude of the plasma generated perturbed parallel current density, shown in Fig. 7(e) and (f), respectively. The coupled case results in about 3 times larger current amplitude, thus stronger screening as shown in Fig. 5 and 6.

\section{Conclusion and discussion}

The aim of this work is a computational understanding of the plasma induced screening effect on the externally applied 3D resonant magnetic field perturbations, in a situation when the plasma flow becomes small, or even vanishes, at one rational surface of the perturbation. The modelling is carried out using the MARS-F code, which incorporates the single fluid, full MHD, resistive plasma model, with full toroidal geometry. We have considered three families of toroidal flow profiles, representing three typical situations of how the flow speed can approach zero near the rational surface. The key physics here is that the presence of a finite, but very slow flow changes the screening regime within the single fluid MHD theory - from the conventional resistive-inertial type of screening to the so called favorable averaged toroidal curvature induced GGJ screening. 
We performed a comprehensive investigation of the subtle and non-trivial screening physics associated with the GGJ effect, in the presence of toroidal flow and flow shear. We find that the local flow amplitude, not the local flow shear, at the rational surface, that is more important in determining the GGJ screening. In addition, the global flow profile also affects the GGJ screening, despite the fact the screening eventually occurs near the rational surface.

An important observation is the nearly perfect cancellation effect between the induction term and the resistive term in Ohm's law, when the GGJ physics is in operation. This is identified as the key physics of the GGJ screening, since the near cancellation between these two terms results in very small amplitude of the resonant radial magnetic field perturbation. On the other hand, such a cancellation does not occur for the RI regime, where the induction term contribution is very small compared to that of the resistive term. These two regimes also significantly differ in terms of the plasma generated screening current. The conventional resistive layer model, which is valid at relatively fast flow, results in a shielding current that peaks at the rational surface, whilst the GGJ model (valid at slow flow) yields a double-peak structure of the perturbed parallel current density along the plasma minor radius. These two peaks occur close but off the rational surface.

We also find that the standard resistive MHD model has an inherent defect in describing the situation where the plasma flow speed vanishes, with the reversal of the flow direction, exactly at the radial location of the perturbation rational surface. Such a degenerated situation leads to un-physical singularity in the plasma response solution. In this study, this degeneracy is resolved by slightly separating the zero-flow surface and the rational surface, and by introducing a peculiar plasma resistivity radial profile, which vanishes at the rational surface, the latter can be viewed as a straightforward, but not unique, way of introducing additional physics into the resistive MHD model without changing the equations. A better model may be obtained by introducing new terms into the equation, such as the hyper-resistivity term in 
Ohm's law [38]. We also point out that no such un-physical singularity is numerically found when the flow speed vanishes at the rational surface while keeping unidirectional flow, as described by our second flow profile model.

The GGJ induced screening may offer a possible interpretation of an interesting observation in the mode locking experiments carried out in TEXTOR [39], where the threshold amplitude of the dynamic ergodic divertor (DED) current, to induce the mode locking, is experimentally determined while scanning the toroidal flow speed of the plasma in both directions. It was found that a finite minimum DED current threshold is required for the mode locking, even at zero flow speed at the rational surface. The single fluid theory without the GGJ screening always predicts zero DED current thresholds at vanishing flow (corresponding to full penetration). The inclusion of the electron diamagnetic flow cannot offer the explanation either, since this only shifts the location of the minimum DED current, without changing the fact of the existence of the finite current threshold.

The results from this study also predict that, with the right conditions (e.g. the plasma does enter into the GGJ screening regime), a low torque regime (thus slow plasma flow) does not necessarily mean a better ELM suppression regime using the RMP fields. This is because the GGJ screening at slow flow may actually prevent the penetration of the RMP field. On the other hand, a slow flow regime may not always be easy to achieve in present day tokamak devices, although it is certainly possible for the toroidal flow to transiently vanish, locally near a rational surface. In ITER, it is likely that slow flow regime will be more easily to access.

Finally, we emphasize that the single fluid model certainly does not contain all the relevant screening physics, in particular the electron diamagnetic flow and the effects of the 
(anisotropic) thermal transport terms near rational surfaces [40, 41]. These remain the topics of our future investigation.

\section{Acknowledge}

The work was partially supported by the National Natural Science Foundation of China (Grant No. 11405029, 11275047, 11505021). This project has also received funding from the European Union's Horizon 2020 research and innovation programme under grant agreement number 633053 and from the RCUK Energy Programme [grant number EP/I501045]. The views and opinions expressed herein do not necessarily reflect those of the European Commission.

\section{References}

[1] Boozer A H 2001 Phys. Rev. Lett. 865059

[2] Liu Y Q 2006 Plasma Phys. Control. Fusion 48969

[3] Lanctot M et al 2010 Phys. Plasmas 17030701

[4] Lanctot M et al 2011 Phys. Plasmas 18056121

[5] Wang Z R et al 2015 Phys. Rev. Lett. 114145005

[6] Liu Y Q et al 2000 Phys. Rev. Lett. 84907

[7] Fransson C M et al 2000 Phys. Plasmas 74143

[8] Bondeson A et al 2001 Nucl. Fusion 41455

[9] Liu Y Q et al 2004 Nucl. Fusion 44232

[10] Chu M S et al 2010 Plasma Phys. Control. Fusion 52123001

[11] Liu Y Q et al 2010 Plasma Phys. Control. Fusion 52104002

[12] Fitzpatrick R et al 1991 Phys. Fluid B 3644

[13] Fitzpatrick R 1998 Phys. Plasmas 53325

[14] Buttery R J et al 1999 Nucl. Fusion 391827 
[15] Park J-K et al 2011 Nucl. Fusion 51023003

[16] Liu Y Q et al 2014 Plasma Phys. Control. Fusion 56104002

[17] Evans T E et al 2006 Nat. Phys. 2419

[18] Liang Y et al 2007 Phys. Rev. Lett. 98265004

[19] Kirk A et al 2011 Plasma Phys. Control. Fusion 53065011

[20] Suttrop W et al 2011 Phys. Rev. Lett. 106225004

[21] Troyon F et al 1984 Plasma Phys. Control. Fusion 26209

[22] Yu Q et al 2008 Nucl. Fusion 48024007

[23] Nardon E et al 2010 Nucl. Fusion 50034002

[24] Liu Y Q et al 2010 Phys. Plasmas 17122502

[25] Liu Y Q et al 2011 Nucl. Fusion 51083002

[26] Bécoulet M et al 2009 Nucl. Fusion 49085011

[27] Ferraro N M et al 2012 Phys. Plasmas 19056105

[28] Turnbull A D et al 2013 Phys. Plasmas 20056114

[29] Haskey S R et al 2014 Plasma Phys. Control. Fusion 56035005

[30] Haskey S R et al 2015 Plasma Phys. Control. Fusion 57025015

[31] King J D et al 2015 Phys. Plasmas 22072501

[32] Ryan D et al 2015 Plasma Phys. Control. Fusion 57095008

[33] Heyn M F et al 2008 Nucl. Fusion 48024005

[34] Liu Y Q et al 2012 Phys. Plasma 19072509

[35] Liu Y Q et al 2000 Phys. Plasmas 73681

[36] Kirk A et al 2015 Nucl. Fusion 55043011

[37] Glasser A H et al 1975 Phys. Fluids 7875

[38] Xu X Q et al 2010 Phys. Rev. Lett. 105175005

[39] Koslowski H R et al 2006 Nucl. Fusion 46 L1

[40] Lutjens H et al 2001 Phys. Plasma 84267 
[41] Connor J W et al 2015 Plasma Phys. Control. Fusion 57065001 